\title{
NEWTONIAN IMPERIALIST COMPETITVE APPROACH TO OPTIMIZING OBSERVATION OF MULTIPLE TARGET POINTS IN MULTISENSOR SURVEILLANCE SYSTEMS
}

\author{
A. Afghan-Toloee ${ }^{\text {a* }}$, A. A. Heidari ${ }^{\text {a }}$, Y. Joibari ${ }^{\text {a }}$ \\ ${ }^{a}$ Dept. of Geomatic Engineering, University of Tehran, Amir-Abad Street, Tehran, Iran \\ - (a.afghantoloee, as_heidari, y.joibari)@ut.ac.ir
}

KEY WORDS: Newtonian imperialist competitive algorithm, multi-sensor, multiple target, collective intelligent, deployment

\begin{abstract}
:
The problem of specifying the minimum number of sensors to deploy in a certain area to face multiple targets has been generally studied in the literatures. In this paper, we are arguing the multi-sensors deployment problem (MDP). The Multi-sensor placement problem can be clarified as minimizing the cost required to cover the multi target points in the area. We propose a more feasible method for the multi-sensor placement problem. Our method makes provision the high coverage of grid based placements while minimizing the cost as discovered in perimeter placement techniques. The NICA algorithm as improved ICA (Imperialist Competitive Algorithm) is used to decrease the performance time to explore an enough solution compared to other meta-heuristic schemes such as GA, PSO and ICA. A three dimensional area is used for clarify the multiple target and placement points, making provision $x, y$, and $z$ computations in the observation algorithm. A structure of model for the multi-sensor placement problem is proposed: The problem is constructed as an optimization problem with the objective to minimize the cost while covering all multiple target points upon a given probability of observation tolerance.
\end{abstract}

\section{INTRODUCTION}

The problem of specifying the minimum number of sensors to deploy in a certain area to face multiple targets has been generally studied in the literatures (J. O'Rourke, 1987). The base of the problem is to use the minimum number of keep safe to observe a polygon area. Each secure can observe any point in its line of sight. In this paper, we are arguing the multi-sensors deployment problem (MDP). The area to be detected is defined by a set of multiple target points to observe. Multi-sensors substitute for the concept of secure. The binary model is defined to survey the MDP that minimize costs to covering the multiple targets (B. Carter, R. Ragade, 2008). The probabilistic model is used in MDP problem because of multisensor will lose the information with distance and environmental conditions. Each sensor can observe any target point within its probabilistic sensing range model. Sensors can be placed at clarified deployment multiple targets in the area. The Multi-sensor placement problem can be clarified as minimizing the cost required to cover the multi target points in the area. A structure of model for the multi-sensor placement problem is proposed: The problem is constructed as an optimization problem with the objective to minimize the cost while covering all multiple target points upon a given probability of observation tolerance.

A probabilistic observation matrix is clarified to separate the observation computation method from the model. This recognizes structure to be used for a broad range of multisensor placement problems. This matrix is defined with the observation probabilities between multi-sensors and multiple targets.
In this paper, the improved ICA (Imperialist Competitive Algorithm) is regarded as the optimization method for deployment the multi-sensor for optimal surveillance the multiple targets. We used collective intelligent in assimilation of colonies to enhance the efficiency of the algorithm. The NICA algorithm is used to decrease the performance time to explore an enough solution compared to deterministic and other base meta-heuristic schemes.

In recent years, many endeavors have been immolated in the research of optimization problems and evaluating the authority of solving the complex problems (Q. Wu et al, 2007). However, most studies were implemented in statistical analysis, rather than theoretical analysis. Although the NICA is more efficiently than ICA, the parameters should be adapted to assurance its optimal solutions.

The remainder of this paper is organized as follows. In Section 2 Imperialist Competitive Algorithm is introduced. Section 3 presents definition of NICA and convergence analysis of NICA with genetic algorithm, PSO and ICA. Section 4 presents model of MDP with probabilistic observation matrix and define fitness of function with this problem. Section 5 contains the experimentations and results. Finally, Section 6 concludes the paper with discussion of the results.

\section{IMPERIALIST COMPETITIVE ALGORITHM}

The global optimization problem is suitable in every field of geomatics. Up until now, many evolutionary algorithms (EA) have been recommended for finding the answers of global optimization problem. Recently, ICA method is offered based on a socio-politically strategy.

\footnotetext{
* Corresponding author
} 
ICA breaks initial population into various sub-populations, and then searches for the optimal answer using two operations of assimilation and competition. The assimilation operation moves each colony in a group toward the best solution (called imperialist) in the same group (Gargari, 2007)

This algorithm starts with random initial solutions. Each unique agent of an empire is called a country, and the colonies and imperialist collectively form empires. Imperialistic competitions between these empires will be the entity of the ICA. During this competition, weak empires breakdown and forceful ones take ownership of their colonies. Imperialistic competitions direct the search procedure in the direction of the optimum solutions.

\section{NEWTONIAN IMPERIALIST COMPETITIVE ALGORITHM}

We want to improve the assimilation operator and movement of countries in ICA. Test done using a set of famous functions. Efficiency of our proposed algorithm proved. Two common Properties of the swarm-based methods are exploration and exploitation. The exploration is ability to search the space, where the exploitation is the ability to hunt the optimum (Gargari, 2007). The exploration is a significant theme in swarm-based heuristic algorithms.

In first iterations, to escape trapping in local optimum, the algorithm must use the exploration to explore the space and find new points. Over time, exploration will be reduced and exploitation fades in, therefore the algorithm adjusts itself in the semi-optimal points. To have a proper search, an important way is a suitable balance between exploration and exploitation. All the swarm-based algorithms use exploration and exploitation, but they use various methods and operators. In other words, all heuristic algorithms have a unique concept.

ICA is unable to execute global search properly in the big problem spaces. During the search process, ICA may trap into local optimum. This causes the bad convergence.

In this paper, a new method is proposed which balances the exploration and exploitation of NICA using colonies powers information.

In the absorption policy step of ICA algorithm, the colonies move in the direction of imperialists with random angles. The colonies motion has a monotonic behavior because of the constant parameter; indeed, the swarm movement could not be changed with search process. Therefore, if the algorithm traps in the local optimum, it cannot leave the trap and move towards the global optimum. To balance between the explorative and exploitative search, gravity between countries is defined and the movement of colonies to the imperialists is adjusted during the search procedure.

Newton's gravitational force is based on acting from distance. This means gravity acts between separated particles on time. In the Newton law, each mass attracts other particles with a force. In ICA, population move to the imperialist by a random deviation. In order to enhance this operator, we offer a Newtonian absorption policy among all solutions. The idea is that neighboring countries have a tendency to improve their political relations. When countries have consensus, weaker countries are more impressionable, based on their proportion with other nations. The countries have less political power, conjunct with their neighbor determinant countries. Political decisions for weaker countries are based on international Political agreements of their Allied nations.
In order to model international relationships for ICA, a pervasive absorption charge is proposed among all countries. The consequent force applied on each colony by its imperialist and all other local colonies of that imperialist would be calculated by considering the power of countries.

In the NICA, all the countries attract to others based on their powers, by the gravitational force, and this force causes a global movement of all nations towards the countries with more power. Hence, countries work together using international communication, modeled by gravitational force. The powerful countries (good solutions) move slower than weaker ones. This promises the exploitation of the algorithm. The absorption charge is defined as follow (eq.1).

$E_{i j}=\zeta \frac{C_{a j} \times C_{p i}}{D^{2}}$,

We have a swarm with $N$ countries. The position of the $i^{\text {th }}$ country $\left(X_{i}\right)$ is defined by equation (2).

$$
X_{i}=\left(\text { country }_{i}, \ldots, \text { country }_{N}, \text { imperialit }_{d}\right)
$$

Where country $_{i}$ is the position of $i$ th country and imperialist $t_{d}$ is the position of $d^{\text {th }}$ imperialist, respectively. At a specific time ' $t$ ', we define the absorption acting on country ' $i$ ' from country ' $j$ ' as equation (3).

$$
E_{i j}^{d}(t)=\zeta(t) \frac{C_{p i}(t) \times C_{a j}(t)}{D_{i j}(t)+\varepsilon}\left(\operatorname{countr}_{j}(t)-\text { country }_{i}(t)\right)
$$

where $C_{a j}$ is the power of country $j, C_{p i}$ is the power related to country $i, \xi(t)$ is absorption constant, $\varepsilon$ is a small constant, and $D_{i j}(t)$ is $2 \mathrm{D}$ distance between two countries $i$ and $j$, calculated according to equation (4)

$D_{i j}(t)=\left\|X_{i}(t), X_{j}(t)\right\|_{2}$

To give a stochastic characteristic to NICA algorithm, total force is randomly weighted sum of the forces of others (equation 5).

$E_{i}^{d}(t)=\sum_{j=1}^{N} \operatorname{rand}_{j} E_{i j}^{d}(t)$

Where $\operatorname{rand}_{j}$ is between $[0,1]$. Hence, the acceleration of the country $i$ at time $t$, and in direction $d^{\text {th }}$, is:

$a_{i}^{d}(t)=\frac{E_{i}^{d}(t)}{C_{i i}(t)}$

Where $C_{i i}$ is the Power of $i^{\text {th }}$ country, the next velocity of country is considered as follows. Therefore, position and its velocity is calculated based on equations (7) and (8).

$$
\begin{aligned}
& v_{i}^{d}(t+1)=\operatorname{rand}_{i} \times v_{i}^{d}(t)+a_{i}^{d}(t) \\
& \text { country }(t+1)=\text { country }(t)+v_{i}^{d}(t+1)
\end{aligned}
$$

Where randi is in [0,1]. This random number is for randomization of the search.

When countries developed, colonization policy will be weaker. To attenuate political dependence over the time, a gravitational constant is initialized at first generation of algorithm and will 
be monotony reduced with time to control the search accuracy. see equation (9)

$$
\zeta(t)=\zeta\left(t_{0}\right) \times\left(\frac{t_{0}}{t}\right)^{\alpha}, \alpha<1
$$

Gravitational and inertia powers are simply calculated by the fitness evaluation. A powerful country means a more efficient nation. This means that better countries have more international relationships and grow more slowly. The gravitational and inertia powers are equal; the powers are calculated by their fitness. We update the Newtonian and inertial powers by the equations (11) and (12).

$$
\begin{aligned}
& C_{a i}=C_{p i}=C_{i i}=C_{i}, \quad i=1,2, \ldots, N \\
& c_{i}(t)=\frac{\operatorname{fit}_{i}(t)-\operatorname{weak}(t)}{\operatorname{strong}(t)-\operatorname{weak}(t)} \\
& C_{i}(t)=\frac{c_{i}(t)}{\sum_{j=1}^{N} c_{j}(t)}
\end{aligned}
$$

Where $f i t_{i}(t)$ shows the fitness of the country $i$ at time $t$, and weak $(t)$ and strong $(t)$ are : (for a minimization problem)

$$
\begin{aligned}
& \text { strong }(t)=\min \text { fit }_{j}(t), \quad j \in\{1, \ldots, N\} \\
& \operatorname{weak}(t)=\max \text { fit }_{j}(t), \quad j \in\{1, \ldots, N\}
\end{aligned}
$$

To avoid local optimum, the algorithm must use the exploration at beginning. The principle of NICA is shown in Figure 1.

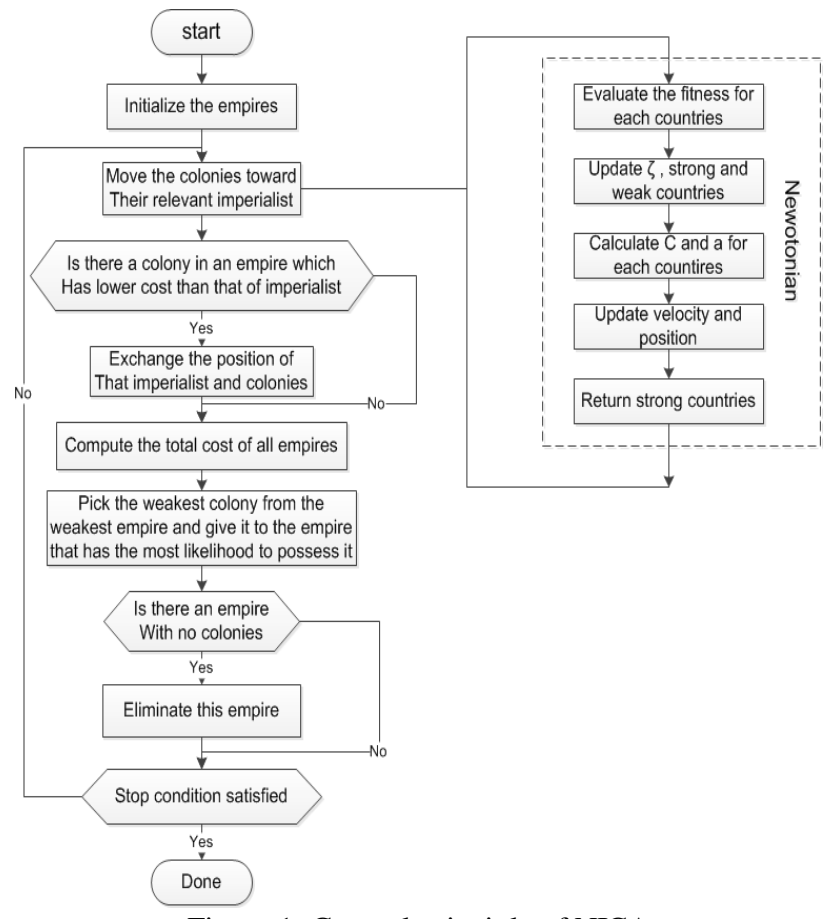

Figure 1. General principle of NICA

\subsection{Analysis and consideration of empirical results}

In this paper, the proposed algorithm, called Newtonian Imperialist Competitive Algorithm (NICA), applied to some famous functions to prove the NICA algorithm performance and compared with ICA and PSO and GA algorithms. The mathematical form and graphical drawings of benchmark functions are represented in Table1 and Figure 2, respectively.

\begin{tabular}{ll}
\hline Mathematical representation & Range \\
\hline$F_{1}(x, y)=\left(x^{2}+y^{2}\right)^{0.25} \times \sin \left\{30\left[(x+0.5)^{2}+y^{2}\right]^{0.1}\right\}+|x|+|y|$ & {$[-10,10]$} \\
$F_{2}(x, y)=J_{0}\left(x^{2}+y^{2}\right)+0.1|1-x|+0.1|1-y|$ & {$[-10,10]$} \\
$F_{3}(X)=\sum_{i=1}^{n}\left(\left|x_{i}\right|-10 \cos \left(\sqrt{\left|10 x_{i}\right|}\right)\right)$ & {$[-10,10]$} \\
$F_{4}(x, y)=x \sin (4 x)+1.1 y \sin (2 y)$ & {$[-10,10]$} \\
\hline
\end{tabular}

Table 1.Benchmark functions

Algorithmic parameters for all algorithms are illustrated in Table 2.

\begin{tabular}{clrlllll}
\hline ICA & \multicolumn{3}{c}{ NICA } & & PSO & & GA \\
\hline Parameter & Value & Parameter & Value & Parameter & Value & Parameter & Value \\
$\mathrm{N}_{\text {pop }}$ & 100 & $\mathrm{~N}_{\text {pop, }}$ & 100 & \#swarm & 100 & Population & 100 \\
$\mathrm{~N}_{\text {imp }}$ & 6 & $\mathrm{~N}_{\text {imp }}$ & 6 & $\mathrm{C}_{1}=\mathrm{C}_{2}$ & 1 & Crossover & 0.7 \\
$\beta$ & 2 & $\xi$ & 0.2 & $\omega_{\min }$ & 0.1 & Mutation rate & 0.2 \\
$\xi$ & 0.2 & $\xi$ & 10 & $\omega_{\max }$ & 0.2 & & \\
$\gamma$ & 0.99 & & & & & & \\
\hline
\end{tabular}

Table 2. parameter values of implemented algorithms

We made simulations for evaluating the speed of convergence and the quality of NICA solution, in comparison to ICA, PSO and GA algorithms. All the benchmarks tested by 10 dimensions separately. The average of optimums for 20 runs obtained.

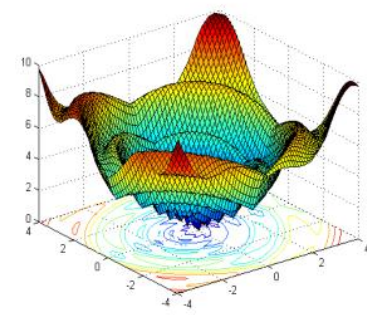

(a)

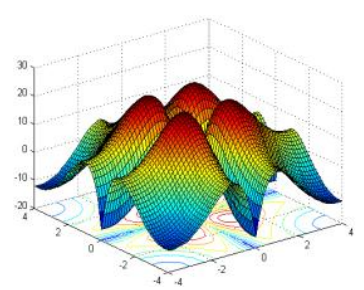

(c)

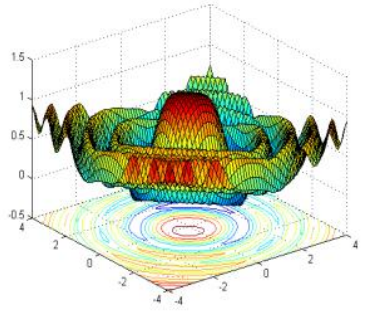

(b)

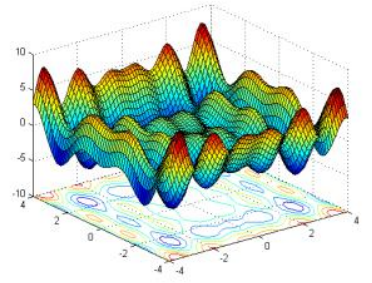

(d)
Figure 2. Visualization of benchmark functions (a) $F_{1}$, (b) $F_{2}$, (c) $F_{3}$, and (d) $F_{4}$

In Figure 3, belongs to $F_{1}$, it is seen that the convergence speed to the optimal point and the quality of global optima solution has improved in compare with three other algorithms. In the plot of the $F_{1}$, at the first 5 iterations, NICA algorithm has better convergence speed than the GA, ICA and PSO and then NICA won the competition. 


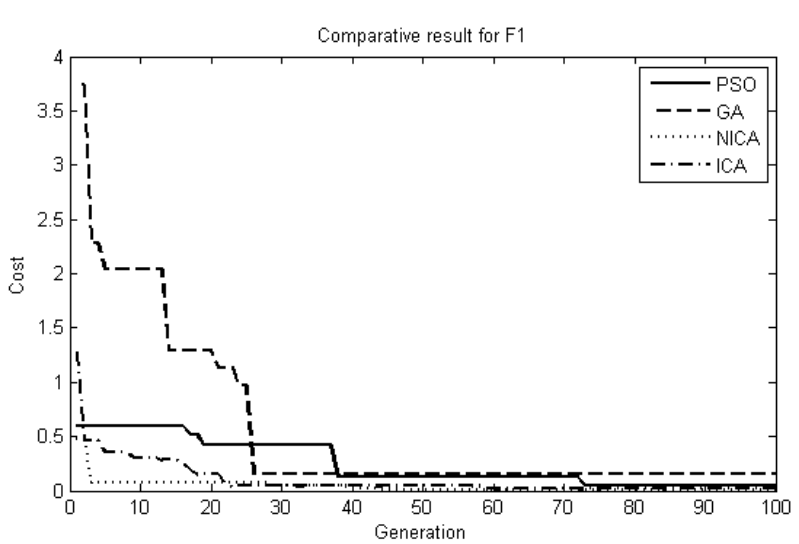

Figure 3. Comparison of performance of NICA, PSO and GA for minimization of $\mathrm{F}_{1}$

The good convergence rate of NICA could be deduced from Figure 4; NICA wants to find the global optimum faster than others and hence has a higher convergence rate.

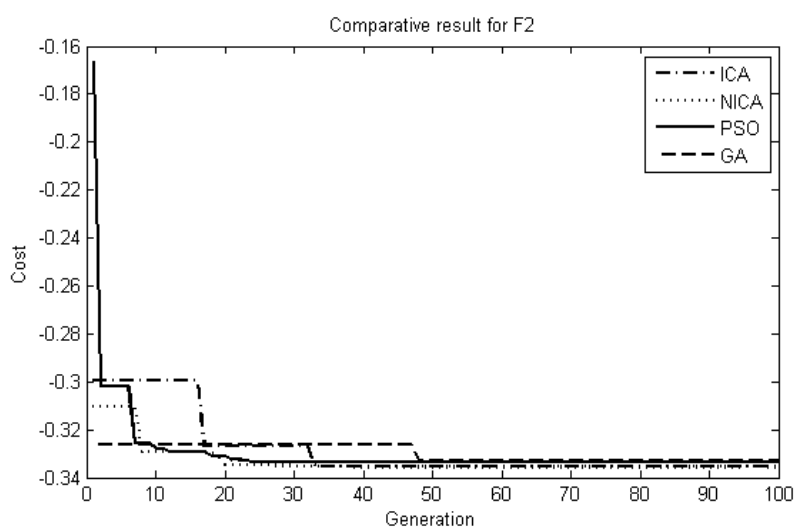

Figure 4. The cost of F2 function

NICA has a good performance for F2 function and can escape from the local peaks and reach to global optimum.

In Figure 5, NICA has remarkable results both in optima solution quality and in convergence.it is seen that the NICA exploration is less than exploitation power for this function.

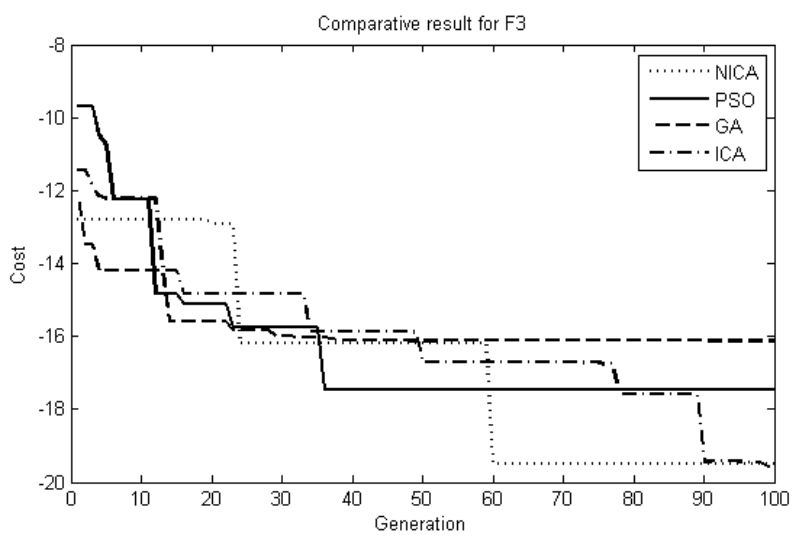

Figure 5. The cost of F3 function.

In Figure 6, F4 function, NICA has better performance in solution quality and in convergence speed rather than PSO and GA algorithms, but NICA curve is near to ICA. At this test in 22th iterations, PSO trapped in local optimum.

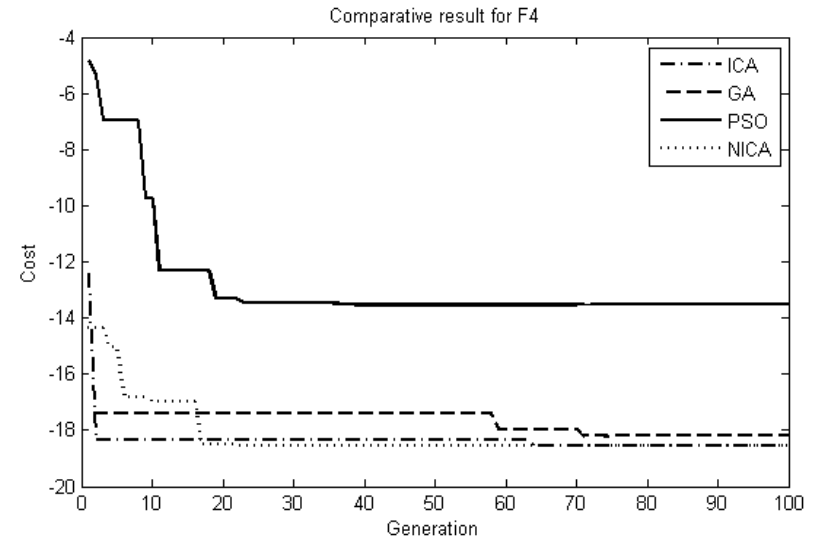

Figure 6. The cost of F4 function.

\begin{tabular}{llcccc}
\hline & & ICA & NICA & PSO & GA \\
\hline $\mathrm{F}_{1}$ & Averagebest-so-far & $2.4 \times 10^{-3}$ & $2 \times 10^{-3}$ & $3.2 \times 10^{-3}$ & 0.252 \\
& Median best-so-far & $1.9 \times 10^{-3}$ & $1.2 \times 10^{-3}$ & $2.8 \times 10^{-3}$ & 0.231 \\
& Averagemean fitness & $3 \times 10^{-3}$ & $2.6 \times 10^{-3}$ & $3.9 \times 10^{-3}$ & 0.326 \\
$\mathrm{~F}_{2}$ & & & & \\
& Average best-so-far & $-31.3 \times 10^{-2}$ & $-33.6 \times 10^{-2}$ & $-30.6 \times 10^{-2}$ & $-28.0 \times 10^{-2}$ \\
& Median best-so-far & $-31.6 \times 10^{-2}$ & $-32.8 \times 10^{-2}$ & $-31.2 \times 10^{-2}$ & $-29 \times 10^{-2}$ \\
& Averagemean fitness & $-31 \times 10^{-2}$ & $-34.2 \times 10^{-2}$ & $-29.7 \times 10^{-2}$ & $-26.8 \times 10^{-2}$ \\
$\mathrm{~F}_{3}$ & Average best-so-far & -19.45 & -19.51 & -17.5 & -16.10 \\
& Median best-so-far & -19.67 & -19.73 & -17.83 & -16.23 \\
& Averagemean fitness & -19.33 & -19.42 & -19.39 & -15.86 \\
$\mathrm{~F}_{4}$ & & & & \\
& Averagebest-so-far & -18.19 & -18.13 & -13.70 & -17.82 \\
& Median best-so-far & -18.29 & -18.23 & -13.82 & -17.92 \\
& Averagemean fitness & -18.13 & -18.7 & -13.63 & -17.76 \\
\hline
\end{tabular}

Table 3. Minimization result of benchmark functions in Table 3. Maximum number of iterations $=100$.

\section{MODEL OF MDP}

\subsection{MDP definition}

In our research, we found that the approaches used to specify observation. Specifying if a multiple target point is observed by a given multi-sensor is dependent on the placement point, orientation, environmental parameters, and other qualities. We approach this by separating the factors from the MDP-Model by defining a probabilistic observation matrix. Using the probabilistic observation matrix $a_{i j}$, we define each row $i$ as a $3 \mathrm{~d}$ grid point $t_{\mathrm{i}}$ which must be observed by at least one sensor and must be observed above a given probability of detection threshold. Each column $\mathrm{j}$ is defined by a deployment multisensor $\mathrm{d}_{\mathrm{j}}$, which applies sensor type and deployment multisensor. Additional qualities may be added to the deployment multi-sensor. Given $a_{i j}=0.90$, demonstrates target $t_{i}$ (row) is observed by deployment multi-sensor $d_{j}$ (column) at a probability of detection of 90 percent where $d_{j}$ defines with a particular multi-sensor type which is deployed at a specific point. Probability of detecting a target is given by following equation.

$$
P_{d}\left(a_{i}\right)=1-\prod_{a_{i j} \in D}\left(1-p\left(a_{i j}\right)\right)
$$

\subsection{Fitness function}

In the MDP-problem the objective is to minimize the cost and maximize the observation. The number of sensors and type of sensors affect on cost and observation. The multi-sensor deployment problem is the problem of observing with 
observation matrix. Defining $x_{j}=1$ if column $\mathrm{j}$ is used in any rows of the solution and $\mathrm{x}_{\mathrm{j}}=0$ otherwise. The cost of deploying column $\mathrm{j}$ is defined as $\mathrm{C}_{\mathrm{j}}$. The multi-sensor deployment problem can be formulated as an optimization problem:

$\min \quad F=k_{1} f_{1}+k_{2} f_{2}+k_{3} f_{3}+k_{4} f_{4}$

$f_{1}$ is the operator for minimum the cost. $f_{2}$ is the punishment of minimum observation and $f_{2}$ is punishment of minimum probability of detecting a multiple target. The $\mathrm{m}$ is the number of multiple targets.

$f_{1}=\sum_{j=1}^{n} C_{j} x_{j}$

$f_{2}=m-$ (number of covered multiple targets)

if $\sum_{j=1}^{n} C_{j} x_{j} \leq 1, \quad f_{3}=f_{3}+1$

if $P_{d}\left(a_{i}\right) \leq \beta, \quad f_{4}=f_{4}+1$

$x_{j} \in\{0,1\}$,

initial solution

\subsection{Case study}

We used the multi-sensor for detection the deformation of the Eiffel tower in Paris that is depicted in figure 7. In this study the multiple targets are points on Eiffel structure and multisensor detect deformation with these points. We design this configuration with Google sketch up software.

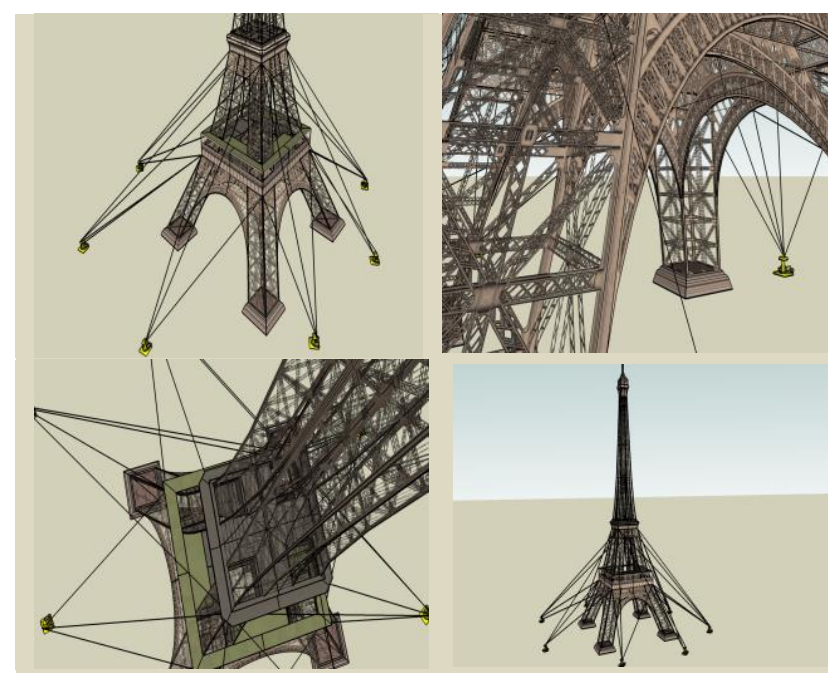

Figure 7. Illustration of the configuration space

The object is to choose a subset of the deployment multi-sensor which minimize cost subject to observing all multiple targets above the given observation threshold. We shall suppose that the observation specification method is defined using a prestructured field of view approach (P. C. F.Y.S. Lin, 2005; J. Adriaens, 2006). To minimize the number of deployment multi-sensor to exhibit, it is supposed that the sensors are fixed and have only one orientation. The observation matrix in this study is defined as follows:

$$
a=\left[\begin{array}{cccccc}
0.90 & 0.90 & 0.80 & 0.90 & 0.90 & 0 \\
0 & 0 & 0 & 0 & 0 & 0 \\
0 & 0 & 0.90 & 0 & 0.80 & 0 \\
0.80 & 0.87 & 0 & 0 & 0 & 0.86 \\
0 & 0.91 & 0.89 & 0.80 & 0.90 & 0 \\
0.87 & 0 & 0.95 & 0 & 0 & 0.87 \\
0.85 & 0 & 0.76 & 0.81 & 0.88 & 0.91 \\
0 & 0.89 & 0 & 0.87 & 0 & 0.95
\end{array}\right]
$$

The cost of the multi-sensor in this study is equal. Therefor the $\mathrm{C}$ is the ones matrix that the size of matrix is the number of multi-sensor. The $\beta$ in this case study is considered 0.15 .

\section{SIMULATION RESULTS}

The convergence speed and amount of objective function in this problem for the Eiffel structure were compared between the NICA, ICA, PSO and GA in Table 4.

\begin{tabular}{|c|l|l|l|l|}
\hline $\begin{array}{l}\text { MDP } \\
\text { problem }\end{array}$ & algorithms & mean & St.dev & $\begin{array}{l}\text { Time } \\
(\mathrm{s})\end{array}$ \\
\hline \multirow{2}{*}{$\begin{array}{c}\text { Control } \\
\text { the Eiffel } \\
\text { structure }\end{array}$} & PSO & 40.23 & 0.185 & 2.6 \\
\cline { 2 - 5 } & GA & 52.34 & 0.112 & 3 \\
\cline { 2 - 5 } & NICA & 32.13 & 0.031 & 1.3 \\
\hline
\end{tabular}

Table 4. Comparative results

For this problem, the optimal solution determined from the algorithms can be supported definitely by generating all the possible permutations. We used the permutations to evaluate the results from Eiffel structure case study which performs the MDP-Model.

\section{CONCLUSIONS}

In the case of an occurrence, efficient and effective deployments of the available sensing and surveillance equipment must be specified. This assessment may be estimated at regular durations based on alter in the occurrence. Thus, NP-complete problems require an effective deployment model which can be used and adjusted based on parameters. Past problem particular models would need the development of new models, which may not be possible due to time constraints or usability of resources. The model exhibited in this paper address the various complexities through the use of parameters. Most notable is the definition of a probabilistic observation matrix. The matrix provides the capability to contain any composition of multi-sensor types, placement points, and other qualities in the model. The performance time is analyzed with respect to GA and PSO and ICA algorithm that indicates the rate of coverage of NICA is more than the other three approaches. The MDP problem is further complicated when the number of multi-sensor is increased.

\section{REFERENCES}

B. Carter, R. Ragade, 2008. An extensible model for the deployment of non-isotropic sensors. IEEE Sensors Applications Symposium, pp. $22-25$. 
B. Carter, R. Ragade, 2009. A Probabilistic Model for the Deployment of Sensors. Sensors Applications Symposium, 7 12.

E. Atashpaz-Gargari and C. Lucas, 2007. Imperialist Competitive Algorithm: An Algorithm for Optimization Inspired by Imperialistic Competition. IEEE Congress on Evolutionary Computation, pp. 4661-4667

J. O'Rourke, 1987. Art Gallery Theorems and Algorithms. Oxford University Press, NJ.

J. Adriaens, S. Megerian, M. Potkonjak, 2006. Optimal worstcase coveragof directional field-of-view sensor networks. Sensor and Ad Hoc Communications and Networks. 3rd Annual IEEE Communications Society on, IEEE, pp. 336-345.

P. C. F.Y.S. Lin, 2005. A near-optimal sensor placement algorithm to achieve complete coverage-discrimination in sensor networks. IEEE, pp. 43-45.

Q. Wu, N. Rao, X. Du, S. Iyengar, V. Vaishnavi, 2007. On efficient deployment of sensors on planar grid. Elsevier's Computer Communications.

Esmat Rashedi, Hossein Nezamabadi-pour, Saeid Saryazdi, 2009. GSA: A Gravitational Search Algorithm. Information Sciences, pp. 2232- 2248. 